\title{
Cambios Refractivos y de Agudeza visual en pacientes pre y post quirúrgicos de Pterigión
}

\author{
Refractive and Visual Acuity Changes in pre- and post-surgical patients with Pterygium \\ Mudanças Refrativos e de Acuidade visual em pacientes pré e pós cirúrgicos de Pterigión
}

Mónica del Rocío Gallegos Murillo

monica.gallegos@cordillera.edu.ec

ORCID: 0000-0003-3050-2530

Instituto Tecnológico Universitario Cordillera, Ecuador

Recibido 21 de octubre 2020 | Arbitrado y aceptado 12 de noviembre 2020 | Publicado 22 de diciembre 2020

\begin{abstract}
RESUMEN
El tratamiento quirúrgico del pterigión es uno de los procedimientos más realizados en la consulta oftalmológica, aunque con frecuencia conlleva molestias y cambios en la agudeza visual. Objetivo: evidenciar si existen cambios en cuanto a agudeza visual y su ametropía, en pacientes diagnosticados con pterigión intervenidos quirúrgicamente, en la ciudad de Quito. Método: se trata de un estudio observacional longitudinal que incluyó 161 pacientes. Se comparó agudeza visual y ametropía antes y 15 días después de la intervención quirúrgica. Resultados: la mayor parte de los pacientes fue de género femenino $(n=118,73 \%)$ tuvo entre 30 y 59 años $(n=127,79 \%)$, presentó pterigión grado III $(n=113,70 \%)$ y fue intervenido del ojo derecho $(n=89,55 \%)$. La agudeza visual de los pacientes mejoró ligeramente. El número de pacientes con agudeza visual 20/20 aumentó de $68(42 \%)$ a $72(45 \%)$. Se observó además una disminución en el número de pacientes con agudeza visual 20/350, 20/200, 20/100, 20/80, 20/60 y 20/50. No hubo cambios en el número de pacientes amétropes que se mantuvo en 69 (43\%). Tanto el número de personas con astigmatismo miópico compuesto, como con astigmatismo mixto eje contra la regla disminuyeron de $11(7 \%)$ a $8(5 \%)$ y de $24(15 \%)$ a $16(10 \%)$, respectivamente. Conclusión: tanto la agudeza visual como la ametropía de pacientes con pterigión mejoran ligeramente después de recibir tratamiento quirúrgico.
\end{abstract}

Palabras clave: pterigión; agudeza visual; refracción; ametropía
MG: Maestrante en Optometría, Licenciada en Optometría, con Certificaciones de Educación continua de la Universidad de Barcelona, Certificación en redacción científica Universidad Católica. Docente académica de las materias de Anatomía, Fisiología y Semiopatología Ocular por 5 años en el ITSCO, Publicación científica en el área de educación (2018), Ponente área de salud visual Escuela Politécnica del Chimborazo. Optómetra en Hospital Militar por 6 años, Gerente General Optimagen por 5 años hasta la actualidad. Instituto Tecnológico Universitario Cordillera, Ecuador.

\begin{abstract}
The surgical treatment of the pterygium is one of the most performed procedures in the ophthalmologic consultation, although it often involves discomfort and changes in visual acuity. Objective: to show if there are changes in visual acuity and its ametropia, in patients diagnosed with surgically intervened pterygium, in the city of Quito. Method: This is a longitudinal observational study that included 161 patients. Visual acuity and ametropia were compared before and 15 days after surgery. Results: most of the patients were female ( $n=118,73 \%)$, were between 30 and 59 years old $(n=127,79 \%)$, presented grade III pterygium ( $n=113,70 \%)$ and was operated on the right eye ( $n=89,55 \%)$. The visual acuity of the patients improved slightly. The number of patients with visual acuity $20 / 20$ increased from $68(42 \%)$ to $72(45 \%)$. There was also a decrease in the number of patients with visual acuity $20 / 350,20 / 200,20 / 100,20 / 80,20 / 60$ and $20 / 50$. There were no changes in the number of ametropic patients that remained in $69(43 \%)$. Both the number of people with compound myopic astigmatism, and with mixed astigmatism axis against the rule decreased from $11(7 \%)$ to $8(5 \%)$ and from $24(15 \%)$ to $16(10 \%)$, respectively. Conclusion: both visual acuity and ametropia of patients with pterygium improve slightly after receiving surgical treatment.
\end{abstract}

Key word: pterygium; visual acuity; refraction; ametropia 
MG: Maestrante en Optometría, Licenciada en Optometría, con Certificaciones de Educación continua de la Universidad de Barcelona, Certificación en redacción científica Universidad Católica. Docente académica de las materias de Anatomía Fisiología y Semiopatología Ocular por 5 años en el ITSCO, Publicación científica en el área de educación (2018), Ponente área de área de educación (2018), Ponente área de
salud visual Escuela Politécnica del salud visual Escuela Politécnica del
Chimborazo. Optómetra en Hospital Militar por 6 años, Gerente General Optimagen por 5 años hasta la actualidad. Instituto Tecnológico Universitario Cordillera Cordillera Ecuador.

\section{RESUMO}

O tratamento cirúrgico do pterigion é um dos procedimentos mais realizados na consulta oftalmológica, embora frequentemente implique transtornos e mudanças na acuidade visual. Objetivo: evidenciar se existem mudanças quanto à acuidade visual e sua ametropia, em pacientes diagnosticados com pterigion, operados cirurgicamente, na cidade de Quito. Método: é um estudo longitudinal observacional que incluiu 161 pacientes. Foi comparada acuidade visual e ametropia antes e 15 dias depois da intervenção cirúrgica. Resultados: a maior parte dos pacientes foi de gênero feminino $(n=118,73 \%)$ teve entre 30 e 59 anos ( $n=127,79 \%)$, apresentou pterigião grau III $(n=113,70 \%)$ e foi operado do olho direito $(\mathrm{n}=89,55 \%)$. A acuidade visual dos pacientes melhorou ligeiramente. $\mathrm{O}$ número de pacientes com acuidade visual 20/20 aumentou de $68(42 \%)$ para 72 (45\%). Observou-se também uma diminuição no número de pacientes com acuidade visual 20/350, 20/200, 20/100, 20/80, 20/60 e 20/50. Não houve mudanças no número de pacientes amétropes que se manteve em 69 (43\%). Tanto o número de pessoas com astigmatismo miópico composto, como com astigmatismo misto eixo contra a regra diminuíram de $11(7 \%)$ para $8(5 \%)$ e de $24(15 \%)$ para $16(10 \%)$, respectivamente. Conclusão: tanto a acuidade visual quanto a ametropia de pacientes com pterigião melhoram ligeiramente depois de receber tratamento cirúrgico.

Palavras-chave: pterigión, agudeza visual, refracción, ametropia

\section{INTRODUCCIÓN}

$\mathrm{E}$ l pterigión se define como una degeneración del colágeno de la conjuntiva bulbar interpalpebral, que clínicamente presenta un aspecto triangular, vascularizado, que invade a menudo la córnea, lo que a menudo se asocia a disminución en la agudeza visual. (1). Se denomina pterigión porque en efecto la lesión que consiste en el desarrollo morboso del tejido que hay debajo de la conjuntiva ocular tiene una forma triangular. (2).

Las causas del pterigión han sido base de amplias especulaciones y todavía no es bien entendido el mecanismo de su desarrollo y recurrencia (3). Sin embargo se ha evidenciado que los pacientes que fueron diagnosticados con esta alteración, estuvieron expuestos a alguno de los siguientes factores de riesgo: exposición a la luz solar sin protección y por periodos prolongados, hiposecreción lagrimal e hipersecreción lagrimal evidenciados a través del test de Shirmer, resultados en test de BUT que sugieran mala calidad lagrimal, exposición a partículas irritantes como el polvo, habitar en áreas polvorientas, historia familiar de la enfermedad, edad avanzada. (4).

Debido a que en las primeras fases el diagnóstico de pterigión se asocia con la aparición de pequeñas lesiones de hiperplasia conjuntival, que se por lo general son asintomáticas, el tratamiento inicial que se recomienda consiste en la administración de antinflamatorios no esteroideos, vasoconstrictores y uso de gafas con protección UVA, UVB y UVC para evitar su crecimiento (5). Sin embargo, múltiples investigaciones han demostrado que la extracción del pterigion mediante una intervención quirúrgica es el tratamiento más idóneo y efectivo. Debido a que este tipo de intervención es relativamente poco invasiva, ya que no requiere hospitalización y es de carácter ambulatorio (6). 
La intervención quirúrgica radica en la extracción completa del pterigión, al igual que el tejido inflamatorio que se ubica a nivel de la córnea y del cuerpo del pterigión. $(5,7)$. Inmediatamente se procede a realizar un auto-injerto conjuntival, que consiste en seccionar una capa fina de tejido conjuntival de la parte superior de la conjuntiva por encima de la hendidura palpebral, la misma que va a ser colocada donde se realizó la extracción del pterigión, a manera de barrera para evitar que vuelva a crecer este tejido $(8,9)$.

Estos cambios anatómicos producidos por el crecimiento del pterigión a nivel de conjuntiva y de la córnea, pueden producir variaciones tanto en la agudeza visual del paciente, como en la ametropía existente o inexistente.

La agudeza visual, es la capacidad que tiene del sistema visual para identificar un objeto, letra, número, etc., con parámetros de mínimos visibles, mínimos separables y mínimos reconocibles (10). Para valorar la agudeza visual de un paciente, se debe tener en cuenta varios parámetros como, distancia al test, iluminación, contraste de luz, posición primaria de mirada, test adecuado para el paciente, referente a edad y grado de escolaridad (11). El resultado de esta prueba indica la capacidad que tiene el paciente de observar y discriminar un objeto a la distancia. El desarrollo del pterigión puede disminuir la agudeza visual del paciente.

Por otro lado, la ametropía o defecto refractivo, consiste en el poder refractivo del ojo, en la que, sin acomodar, el punto conjugado de la retina no coincide con el infinito óptico. Ante esta situación la imagen se enfoca delante o detrás del plano de la retina, resultando en visión borrosas (10). Las ametropías esféricas como la miopía y la hipermetropía pueden estar o no relacionadas con el pterigión sin embargo el astigmatismo tiene una relación estrecha, ya que este, está dado por la superficie corneal irregular, que cuando existe una asimetría produce esta condición de Astigmatismo, y el pterigión es un desencadenante para que aparezca dicha ametropía. Por otro lado, la patogénesis de las recurrencias parece ser diferente a la del pterigión. (12), Sin embargo según (13) y varios autores, la cirugía ocular permite corregir este tipo de trastorno.

Para dar una definición óptica de lo que es una ametropía o defecto refractivo, es una condición en la que los rayos de luz paralelos que ingresan al globo ocular se enfocan delante o detrás del plano de la retina produciendo que la imagen de los objetos sea borrosa (14). Mientras que el Astigmatismo produce que la luz que ingresa al ojo se enfoque en diferentes puntos focales. Donde la córnea y las superficies del cristalino son los factores principales que contribuyen que al menos exista un ligero grado de astigmatismo, siendo el astigmatismo corneal, la mayor causa de astigmatismo ocular (15), y es donde el pterigión se va a ubicar.

Si bien el pterigión tiene un tratamiento adecuado como lo es la cirugía, éste está encaminado a la parte del confort del paciente como de su estética, por tanto, El trabajo tiene como objetivo evidenciar y dar a conocer que también se dan, cambios estructurales que pueden disminuir la agudeza visual y cambiar las ametropías existentes o incluso desarrollar astigmatismo que no tenía el paciente antes del desarrollo del pterigión y que una vez que fueron intervenidos quirúrgicamente, estos cambios fueron evidenciados.

Es por tanto importante que cuando se presente los tratamientos adecuados para dicha patología se lo presente no solo desde el plano que se va a poder mejorar la parte estética, sino que existen otras consideraciones que son de igual 
importancia, para que el paciente se pueda someter a la cirugía.

\section{MATERIALES Y MÉTODOS}

$\mathrm{L}$

a investigación fue longitudinal, se valoró a los pacientes en dos tiempos antes de la cirugía y 15 días después de la misma. Los aspectos evaluados en los pacientes fueron su agudeza visual y diagnóstico refractivo pre y post quirúrgico. La información clínica de los pacientes fue recolectada a través de una historia clínica previamente diseñada para esta investigación. La misma que recogía a más de los datos demográficos, los resultados de las pruebas de agudeza visual, refracción, diagnóstico refractivo, entre otros.

\section{Criterios de inclusión}

Pacientes que fueron operados de pterigión, en una entidad pública de la ciudad de Quito, ambos géneros, con datos clínicos optométricos pre y post quirúrgicos.

\section{Agudeza visual}

Se midió la agudeza visual sin corrección (sin de lentes), con corrección (con de lentes correctivos) pre y post quirúrgicas utilizado la tabla de Snellen, donde la mejor agudeza visual es equivalente a 20/20 y valores inferiores como 20/100 sugieren alteraciones de la agudeza visual (16). Para medir la agudeza visual se siguieron los siguientes pasos: a). Sentar de manera cómoda y confortable al paciente, en una habitación con buena iluminación; b). Colocar el optotipo a una distancia de $6 \mathrm{~m}$.; c). Ocluir ojo izquierdo para valorar ojo derecho y viceversa; d). Explicar al paciente que observará una serie de letras, las cuales deberá leerlas en voz alta; e). Pedir al paciente que indique cuando las letras se han vuelto difíciles de identificar; f). Si el paciente ha reportado dificultad en la identificación de la letra, se debe proceder a anotar la agudeza visual en la historia clínica y dar por finalizado la toma de visión en ese ojo, y g). Ocluir ojo izquierdo y repetir los pasos anteriores.

\section{Rinoscopia}

El propósito de la rinoscopia estática fue obtener una medida objetiva del estado refractivo del ojo, mientras el paciente fija un objeto ubicado a 6 metros de distancia, con la acomodación en reposo (17). Con lo que se estableció un diagnóstico refractivo pre y post quirúrgicas. Para esta evaluación se siguieron los siguientes pasos: a). Sentar al paciente apoyando su cabeza sobre el soporte que lleva el espaldar de la silla; b). Ajustar la altura de la silla de tal manera que los ojos del paciente estén a la misma altura del examinador; c). Este procedimiento debe hacerse sin corrección óptica y con ambos ojos abiertos; d). Montura de prueba delante de los ojos del paciente con la correspondiente distancia interpupilar y ajustar el nivel del instrumento centrando los ojos; e). Colocar el lente de relajación ya sea en la montura de prueba o en el forópter (se debe conocer el valor numérico del lente de relajación compensada con la distancia de trabajo); f). Colocar un punto de fijación luminoso a $6 \mathrm{~m}, \mathrm{yg}$ ). Pedir al paciente que mantenga los ojos abiertos y mire el punto de fijación de lejos, durante el procedimiento.

\section{Evaluación del grado de pterigión}

El pterigión puede ser asintomático y producir únicamente alteración estética. Los síntomas más frecuentes suelen ser irritativos, habitualmente leves, como quemazón, enrojecimiento, fotofobia, lagrimeo 
y sensación de cuerpo extraño. La severidad de los síntomas es proporcional al tamaño y morfología (18).

En la evaluación para la clasificación del pterigión, se tomó en cuenta los parámetros antes mencionados, al igual que el grado de invasión y de actividad, así como su localización, como sugiere (19).

La clasificación del pterigión según los siguientes parámetros descriptivos es importante a la hora de tomar decisiones en cuanto a las indicaciones de cirugía como lo es la morfología; invasión corneal; compromiso limbar; grado de actividad; primario/recidivado; lateralidad; polaridad; otras alteraciones asociadas.

\section{RESULTADOS}

$\mathrm{L}$ a muestra incluyó 161 personas de las cualesse obtuvo los siguientes resultados:

\section{Agudeza visual sin corrección}

Los resultados de la agudeza visual sin corrección pre y post quirúrgicos se muestran en la Tabla 1. Se observó que el número de pacientes con agudeza visual 20/20 antes de la cirugía incrementó de 68 $(42 \%)$ a $72(45 \%)$ después de la cirugía. Algo similar ocurrió con el número de pacientes con agudeza visual 20/350, $20 / 200,20 / 100,20 / 80,20 / 60$ y $20 / 50$, que también disminuyó después de la cirugía, aunque ligeramente (Tabla 1). Por otro lado, aunque el número de pacientes con 20/40 no cambió, si lo hizo el número de pacientes con agudeza visual $20 / 30$ y $20 / 25$ pero en sentido contrario. Después de la cirugía este número se incrementó de $18(11 \%)$ a 28 $(17 \%)$ y de $11 \quad(7 \%)$ a 18 (11\%), respectivamente (Tabla 1 ).

Tabla 1. Relación de la agudeza visual sin corrección pre y post quirúrgica del ojo intervenido (27).

\begin{tabular}{ccccccc}
\hline \multicolumn{6}{c}{ RELACION DE LA AGUDEZA VISUAL SIN CORRECCION } \\
PRE QUIRURGICA & PACIENTES & $\%$ & $\begin{array}{c}\text { POST } \\
\text { QUIRURGICA }\end{array}$ & PACIENTES & $\%$ \\
\hline $20 / 350$ & 3 & 1.9 & & $20 / 250$ & 2 & 1.2 \\
& & & $20 / 200$ & 3 & 1.9 \\
$20 / 200$ & 10 & 6.2 & $20 / 150$ & 2 & 1.2 \\
& & & $20 / 100$ & 4 & 2.5 \\
$20 / 100$ & 5 & 3.1 & $20 / 80$ & 8 & 5.0 \\
$20 / 80$ & 10 & 6.2 & $20 / 60$ & 3 & 1.9 \\
$20 / 60$ & 8 & 5.0 & $20 / 50$ & 8 & 5.0 \\
$20 / 50$ & 15 & 9.3 & $20 / 40$ & 13 & 8.1 \\
\hline
\end{tabular}




\begin{tabular}{|c|c|c|c|c|c|}
\hline \multicolumn{6}{|c|}{ RELACION DE LA AGUDEZA VISUAL SIN CORRECCION } \\
\hline PRE QUIRURGICA & PACIENTES & $\%$ & $\begin{array}{c}\text { POST } \\
\text { QUIRURGICA }\end{array}$ & PACIENTES & $\%$ \\
\hline $20 / 30$ & 18 & 11.2 & $20 / 30$ & 28 & 17.4 \\
\hline $20 / 25$ & 11 & 6.8 & $20 / 25$ & 18 & 11.2 \\
\hline $20 / 20$ & 68 & 42.2 & $20 / 20$ & 72 & 44.7 \\
\hline TOTAL & 161 & $100 \%$ & TOTAL & 161 & $100 \%$ \\
\hline
\end{tabular}

\section{Agudeza visual con corrección}

Los resultados de la agudeza visual con corrección pre y post quirúrgicos se muestran en la Tabla 2. Se observó que el número de pacientes con agudeza visual 20/20 antes de la cirugía incrementó de 97
(60\%) a 144 (89\%) después de la cirugía. Algo similar ocurrió con el número de pacientes con agudeza visual 20/200, 20/100, 20/80, 20/60, 20/50, 20/40, 20/30 y $20 / 25$, que también disminuyó después de la cirugía, aunque ligeramente (Tabla 2).

Tabla 2. Relación de la agudeza visual con corrección pre y post quirúrgica del ojo intervenido (27).

\begin{tabular}{cccccc}
\hline $\begin{array}{c}\text { RELACION DE LA AGUDEZA VISUAL CON CORRECION } \\
\text { PRE } \\
\text { QUIRURGICA }\end{array}$ & PACIENTES & $\%$ & $\begin{array}{c}\text { POST } \\
\text { QUIRURGICA }\end{array}$ & PACIENTES & $\%$ \\
\hline $20 / 200$ & 1 & 0.6 & & & \\
$20 / 100$ & 1 & 0.6 & & 2 & 1.2 \\
$20 / 80$ & 3 & 1.9 & $20 / 80$ & & \\
$20 / 60$ & 2 & 1.2 & & & \\
$20 / 50$ & 1 & 0.6 & & 1 & 0.6 \\
$20 / 40$ & 8 & 5.0 & $20 / 40$ & 6 & 3.7 \\
$20 / 30$ & 14 & 8.7 & $20 / 30$ & 8 & 5.0 \\
$20 / 25$ & 34 & 21.1 & $20 / 25$ & 144 & 89.4 \\
$20 / 20$ & 97 & 60.2 & $20 / 20$ & $\mathbf{1 6 1}$ & $\mathbf{1 0 0}$ \\
\hline
\end{tabular}




\section{Ametropía}

Los resultados en cuanto al diagnóstico refractivo pre y post quirúrgico se muestran en la Tabla 3. Se observó una disminución del número de pacientes con astigmatismo miópico compuesto eje contra la regla (AMC EJE AR) que pasó de $11(7 \%)$ a 8 (5\%). Algo similar ocurrió con el número de personas con astigmatismo mixto con eje con la regla (A MIXTO EJE WR), contra la regla (A MIXTO EJE AR) y oblicuo (A MIXTO EJE OBL), que pasó de $29(18 \%)$ a $16(10 \%)$. El número de personas con otros diagnósticos no cambió o aumentó ligeramente (Tabla 3).

Tabla 3. Relación de los diagnósticos pre y post quirúrgicos del ojo intervenido + AMS astigmatismo miópico simple +AMC astigmatismo miópico compuesto +AHS astigmatismo hipermetrópico Simple + AHC astigmatismo hipermetrópico compuesto $+W R$ con la regla+ AR contra la regla+ OBL oblicuo (27).

\begin{tabular}{|c|c|c|c|c|c|}
\hline \multicolumn{6}{|c|}{ RELACIÓN DE LOS DIAGNOSTICOS REFRACTIVOS } \\
\hline $\begin{array}{c}\text { PRE } \\
\text { QUIRUGICO }\end{array}$ & PACIENTES & $\%$ & $\begin{array}{c}\text { POST } \\
\text { QUIRURGICO }\end{array}$ & PACIENTES & $\%$ \\
\hline EMEPTROPE & 69 & 42.9 & EMEPTROPE & 69 & 42.9 \\
\hline AMS EJE WR & 16 & 9.9 & AMS EJE WR & 22 & 13.7 \\
\hline AMS EJE AR & 1 & 0.6 & AMS EJE AR & 3 & 1.9 \\
\hline AMC EJE WR & 16 & 9.9 & AMC EJE WR & 17 & 10.6 \\
\hline AMC EJE AR & 11 & 6.8 & AMC EJE AR & 8 & 5.0 \\
\hline AMS EJE OBL & 1 & 0.6 & AMS EJE OBL & 2 & 1.2 \\
\hline AMC EJE OBL & 3 & 1.9 & AMC EJE OBL & 3 & 1.9 \\
\hline MIOPIA & 1 & 0.6 & MIOPIA & 1 & 0.6 \\
\hline AHS EJE WR & 1 & 0.6 & AHS EJE WR & 1 & 0.6 \\
\hline
\end{tabular}

Si bien los siguientes resultados no fueron objeto de estudio, los datos son relevantes para posteriores estudios por lo que se adjunta dichos porcentajes

\begin{tabular}{cccccc}
\hline $\begin{array}{c}\text { RELACIÓN DE LOS DIAGNOSTICOS REFRACTIVOS } \\
\text { PRE } \\
\text { QUIRUGICO }\end{array}$ & PACIENTES & $\%$ & $\begin{array}{c}\text { POST } \\
\text { QUIRURGICO }\end{array}$ & PACIENTES & $\%$ \\
\hline AHC EJE WR & 7 & 4.3 & AHC EJE WR & 13 & 8.1 \\
AHC EJE AR & 2 & 1.2 & AHC EJE AR & 2 & 1.2 \\
AHC EJE OBL & 2 & 1.2 & AHC EJE OBL & 2 & 1.2 \\
HIPERMETROPIA & 2 & 1.2 & HIPERMETROPIA & 2 & 1.2 \\
A MIXTO EJE AR & 2 & 1.2 & A MIXTO EJE AR & 0 & - \\
A MIXTO EJE WR & 24 & 14.9 & A MIXTO EJE WR & 16 & 9.9 \\
A MIXTO EJE OBL & 3 & 1.9 & A MIXTO EJE OBL & 0 & - \\
\hline
\end{tabular}


Tabla 4. Frecuencia de género (27).

\begin{tabular}{lcc}
\hline & FRECUENCIA & PORCENTAJE \\
\hline MASCULINO & 43 & 26.7 \\
FEMENINO & 118 & 73.3 \\
TOTAL & $\mathbf{1 6 1}$ & $\mathbf{1 0 0}$ \\
\hline
\end{tabular}

Tabla 5. Frecuencia de Edad (26).

\begin{tabular}{lcc}
\hline & FRECUENCIA & PORCENTAJE \\
\hline $20-29$ & 13 & 8,1 \\
$30-39$ & 30 & 33,5 \\
$40-49$ & 46 & 28,6 \\
$50-59$ & 27 & 16,8 \\
$60-69$ & 12 & 7,5 \\
$70-79$ & 7 & 7,3 \\
Más de 80 & 2 & 1,2 \\
TOTAL & $\mathbf{1 6 1}$ & $\mathbf{1 0 0}$ \\
\hline
\end{tabular}

La muestra incluyó 161 personas, de las cuales $43(27 \%)$ fueron de género masculino y 118 (73\%) de género femenino (Tabla 4). De los 161, 159 tuvieron entre 20 y 80 años, solo 2 tuvieron más de 80 años. Al clasificarlos por rangos de edad se observó que 13 pacientes tuvieron entre $20-29$ años, 54 pacientes entre $30-39$ años, 46 pacientes entre 40 - 49 años, 27 pacientes entre 50 - 59 años, 12 pacientes entre 60 69 años, 7 pacientes entre $70-79$ años y 2 pacientes en más de 80 años. La mayor parte de pacientes, lo que corresponde 127 (79\%) tuvieron entre 30 y 59 años (Tabla 5). Según el grado de pterigión, se determinó que 38 (24\%) presentó grado II, $113(70 \%)$ grado III y 10 (6\%) grado IV. (Tabla 6).

Tabla 6. Grado de Pterigión (27)

\begin{tabular}{lcc}
\hline & FRECUENCIA & PORCENTAJE \\
\hline GRADO I & 38 & 23,6 \\
GRADO II & 113 & 70,2 \\
GRADO III & 10 & 6,2 \\
TOTAL & $\mathbf{1 6 1}$ & $\mathbf{1 0 0}$ \\
\hline
\end{tabular}




\section{DISCUSIÓN}

$\mathrm{E}$ l pterigión como se ha mencionado es una patología común, lo que permite tener estudios alrededor sobre esta enfermedad, sin embargo, todos van encaminados a eliminar la sensación de cuerpo extraño en el paciente y mejorar la parte estética, que no tienen una dirección en la parte de agudeza visual o cambio refractivo.

$\mathrm{Si}$ bien en estudios epidemiológicos llevados a cabo por diferentes autores y en distintas regiones del mundo, se describe, una mayor incidencia de pterigión en personas de edad media y avanzada, pero sin predominio de géneros cuando las condiciones de vida son similares. (20). En la investigación la mayoría de los pacientes que presentaron pterigión se encontraba en el rango de edad de 30-39 años (34\%) y de 40 a 49 (29\%), y estos rangos de edad no son considerados una población vulnerable usual para desencadenar esta patología, resultados que puede no coincidir con otros como el llevado a cabo por la Universidad de Cuenca donde describen que "esta patología es más frecuente entre los pacientes de 51 a 60 años. (21). Rango que en el estudio obtuvo únicamente un $7 \%$.

La investigación como tal determinó una relación entre la patología y las ametropías, si bien las ametropías no están relacionadas con alteraciones a nivel de conjuntiva, el pterigión cuando se encuentra en grados avanzados puede alterar la anatomía de la córnea lo que conlleva a la aparición de una ametropía cilíndrica como es el astigmatismo. (19)

En la investigación se pudo obtener una mejoría en la toma de agudeza visual, de al menos 1 a 2 líneas lo que es realmente significativo en cuanto a calidad visual.

En base a este resultado de mejoría de agudeza visual, se determinó que el tipo de pterigión que prevaleció para la cirugía es el de grado III con 113 pacientes intervenidos, lo que corrobora estudios previos que concuerdan con estos resultados.

De acuerdo con los diagnósticos refractivos prequirúrgicos los astigmatismos mixtos con la regla, contra la regla y oblicuos se presentan en 29 casos, los mismos que después del postquirúrgico disminuyen a 16 pacientes.

En la agudeza visual óptima sin corrección prequirúrgica encasillada en $20 / 20$ con 68 pacientes, pasa a 72 pacientes después de la cirugía, mejorando significativamente la agudeza visual de estos pacientes.

La mayoría de las cirugías fueron en el ojo derecho, pero no existe una diferencia significativa con el OI.

\section{Cambios ametrópicos}

De acuerdo con los datos obtenidos en la investigación existen cambios refractivos posquirúrgico, con aumento de la agudeza visual, sin embargo, en estudios anteriores con pterigión sin invasión corneal no fueron evidenciados, por lo tanto, tampoco existirían cambios en la agudeza visual.

\section{Cuidados prequirúrgicos}

Los cuidados preoperatorios de cualquier tipo de cirugía deben estar bien definidos para evitar algún tipo de complicación durante el proceso quirúrgico (22).

Estos deben estar indicados por el especialista o su equipo médico, con la finalidad que, una vez terminada la intervención, se evite molestias e interrogantes que puedan producir ansiedad en el paciente y nos den mejores resultados (23). 


\section{Cuidados post quirúrgicos}

Todo proceso quirúrgico conlleva cuidados post operatorios especiales, para evitar posibles complicaciones y obtener un resultado óptimo.

Los cuidados que debe tener el paciente después de la cirugía, deben ser dados por el cirujano o su equipo médico que lo asistió, como acudir a una valoración refractiva después de la cirugía para evaluar la agudeza visual y las ametropías. $(10,24)$.

\section{Este proceso se puede evitar}

La protección solar que brindan las gafas con filtro ultravioleta permite la prevención del Pterigión, junto con el uso de gorras. En Ecuador, la intensidad de la radiación solar es más intensa entre las 10:00 y las 14:00. Por eso se recomienda protegerse del sol entre esas horas siempre (25).

\section{CONCLUSIONES}

B asado en los resultados alcanzados en la investigación se cumplió con el objetivo de evidenciar que, si existen cambios tanto en la agudeza visual y refracción en los pacientes que son sometidos a cirugía de pterigión, y que dichos cambios son positivos en la calidad de visión del paciente, que, si bien la cirugía está encaminada a la solución de otros problemas que el paciente refiere, estos otros parámetros deben ser tomados muy en cuenta al momento de presentar un tratamiento adecuado como la cirugía.

Entre los cambios positivos post quirúrgico que se pudo evidenciar en el estudio, es la mejoría en la toma de agudeza visual, de al menos 1 a 2 líneas, que es realmente significativo en cuanto a calidad visual, y que los resultados en la agudeza visual óptima sin corrección pre quirúrgica encasillada en $20 / 20$ con 68 pacientes, pasó a 72 pacientes después de la cirugía, concluyendo que la presencia del pterigión si disminuyo la agudeza visual de estos pacientes.

En cuanto a los cambios refractivos, de acuerdo a los diagnósticos pre quirúrgicos los astigmatismos mixtos con la regla, contra la regla y oblicuos que se presentaron estaban en 29 casos, los mismos que después del postquirúrgico disminuyen a 16 pacientes. Por tanto, la alteración producida en la estructura anatómica de la córnea por la presencia del pterigión si produce cambios ametrópicos.

\section{REFERENCIAS BIBLIOGRÁFICAS}

1. Cabrera Silva S, Lissi Gervaso E, Honeyman Mauro J. Radiación ultravioleta y salud Santiago de Chile: Universitaria S.A; 2005

2. Andreq Keirl CC. Clinical Optics and Refraction USA: Elsevier; 2007

3. Espinal Guillen D. Pterigion. Una guía práctica de diagnóstico y tratamiento. Medica hondureña. 1995; p. 102-104

4. Ríos Figueroa MF. Cambios en la agudeza visual, la queratometria y refracción en pacientes después de cirugía de pterigion grado I y grado II. Ciencia Tecnología y Salud. 2012; p. 101 $-108$

5. Barragán JACBMMGBJED. El Pterigion. Salud Bosque. 2011; p. 41-46

6. Boyd S, Gutiérrez AM, Dr. McCulley J. Altlas y texto de Patología y Cirugía Corneal Panamá: Medical Ltd.; 2011

7. Agencia Nacional de Regulación, control y vigilancia sanitaria. Agencia Nacional de Regulación, control y vigilancia sanitaria. [Online]; 2015. Available from: http://www.controlsanitario.gob.ec/wpcontent/uploads/downloads/2014/09/I nstructivo-descriptivo-deestablecimientos-sujetos-a-vigilanciasanitaria.pdf 
8. Diaz DLIL, Quiros DNB, Ramos GV. Tratamiento quirúrgico del Pterigion Primario con autoplastia conjuntival. Archivo Médico de Camagüey. 2010

9. Keyly Fernández Gracia DZGCDACPDZPPDMJODCAPR.

Autoinjerto conjuntival y membrana amniótica en la cirugía del pterigión primario. Cubana de Oftalmología. 2010.

10. Figueroa MFR. Ciencia y Tecnología para la Salud Visual y Ocular. [Online]; 2012. Available from: http://revistas.lasalle.edu.co/index.php /sv/article/view/98/54

11. Collado Hornillos J, Gómez Dacasa A, Caviedes SA. Oftalmología II España: Servicio de Publicaciones: Universidad de Cantabria; 1992

12. Asamblea Constituyente del Ecuador. Régimen del Buen Vivir. 2008 Julio 24

13. Scarlatti D. Consejos para antes y después de la Cirugía Ocular. [Online]; 2012. Available from: http://www.drairadier.com/consejos/c onsejos_cirugia.htm

14. Guerrero J. Optometría Clínica. Seccional ed. Bucaramanga Colombia: Universidad Santo Tomas; 2016

15. Universidad Complutense de Madrid. Óptica Fisiológica: El Sistema óptico del ojo y la visión binocular. [Online]; 2019. Available from: http://eprints.ucm.es/1482/1/Puell \%C3\%93ptica_Fisiol\%C3\%B3gica.pdf

16. Furlan W, Garcia Montereal J, \& Muñoz Escrivá L. Fundaments de Optometría, 2da edición: Refracción Ocular Valencia: Universidad de Valencia; 2011

17. Grosvenor T. Primary Care Optometry, San Luis: Missouri, Fifth edition: Butterworth Heinemann, 2007: 191200

18. Urrutia MTI. Sociedad Española de Oftalmología. [Online]; 2017 [cited 2019
$1012 . \quad$ Available from: https://www.oftalmoseo.com/docume ntacion/nuevos_protocolos/Cirugiadel-pterigium.pdf

19. Cuan Aguilar Yoriel ÁMJMDECDTHPI. Rev Cubana Oftalmol. [Online]; 2016 [cited 201910 12. Available from: http://scielo.sld.cu/scielo.php?script=sc i_arttext\&pid=S0864-

21762016000200011\&lng=es

20. Cameron. Pterygium thoughout the word CC Thomas, editor. Illinois: Springfield; 1965.

21. Espinoza León LA, \& LGHA. Universidad de Cuenca. [Online]; 2018 [cited 2019 10 12]. Available from: http://dspace.ucuenca.edu.ec/handle/1 23456789/31333

22. M R. Cambios en la agudeza visual, la queratometria y refracción en pacientes después de cirugía de pterigion grado I y grado II. Ciencia \& tecnología para la salud visual y ocular. [Online]; 2012. Available from: https://prezi.com/yk_yucvit1mg/como -afecta-el-pterigion-el-sistema-visual/

23. Díaz Alfonso LR, DEMHDHGA, y DJAN. Estudio comparativo de 2 técnicas quirúrgicas para la cirugía del pterigión primario. Cubana de Oftalmología. 2000

24. Medline P. Agudeza Visual. [Online]; 2016. Available from: https://medlineplus.gov/spanish/ency /article/003396.htm

25. Center L. Visión 20/20. [Online]; 2015. Available from: http://es.lasercentervision2020.com/o peracion-pterigium-Quito-Ecuador/8/

26. INEN. Instituto Ecuatoriano de Normalización. 2012 Septiembre 21

27. Espinoza B. Dspace cordillera. [Online]; 2016 [cited 2018. Available from: https://cutt.ly/PlrUwHz 